\section{AB0105 1 A SYSTEMATIC REVIEW OF THE MULTI-BIOMARKER DISEASE ACTIVITY SCORE FOR THE DIAGNOSIS AND TREATMENT IN RHEUMATOID ARTHRITIS}

D. Abdelhafiz ${ }^{1}$, M. Bukhari ${ }^{2} .{ }^{1}$ Lancaster, Lancaster Medical School, Lancaster, United Kingdom; ${ }^{2}$ University Hospitals of Morecambe Bay NHS Trust, Rheumatology, Lancaster, United Kingdom

Background: Biomarkers are important tools that can be used in the diagnosis and monitoring of rheumatoid arthritis (RA). The multi-biomarker disease activity (MBDA) score has shown a clinical value in the overall management of patients with RA.

Objectives: To systematically explore the role of MBDA score for diagnosis and treatment of RA.

Methods: A systematic review was conducted in accordance with the Preferred Reporting Items for Systematic Reviews and Meta-Analyses (PRISMA) recommendations. Key words used included: 'Rheumatoid Arthritis', 'Biomarkers', 'Diagnosis', 'Treatment', 'Outcome', 'Response to treatment', 'Disease Activity', and 'MBDA' The literature search included papers published in 2020 and in the English language.

Results: This literature search has yielded 5 studies that investigated the impact of MBDA score on the diagnosis, treatment or disease progression of RA and have reported clear and clinically validated diagnostic or therapeutic effects. The first study prospectively investigated 130 patients with RA, and has shown that the MBDA score moderately correlated with disease activity at baseline $(r=0.36$ for adjusted MBDA score, $r=0.44$ for unadjusted MBDA score) and at 16 weeks follow up ( $r=0.20$ for adjusted MBDA, $r=0.30$ unadjusted MBDA) but did not predict treatment response to methotrexate. There was also moderate correlation between MBDA scores and erythrocyte sedimentation rate (ESR) at baseline $(r$ $=0.54$ and 0.59$)$ and week $16(r=0.42$ and 0.51$)$. The second was a cross-sectional study, which included 104 patients $(50 \%$ female) and demonstrated that adjusted and unadjusted MBDA score correlated similarly with clinical disease activity. Adjustment for leptin reduced the influence of adiposity, particularly among women but significantly estimated higher disease activity in thin men and women. However, MBDA score predicted disease activity and response to tofacitinib treatment, measured by musculoskeletal ultrasound score (MSUS), in RA patients as demonstrated by the third prospective study that included 25 patients (all $p<0.05$ ). Correlation was found between baseline MSUS and MBDA score, and with 12-week changes in clinical disease activity $(r=0.45-0.62, p<0.05)$. Regression analysis showed associations between baseline MBDA score and 6 -week (all $p<0.05$ ) and 12-week change in change in clinical disease activity $(p=0.03)$. The fourth prospective study which included 92 patients with RA, showed that MBDA could predict response to treatment of methotrexate with or without prednisolone. The improvement was faster in the first month of treatment followed by gradual improvement over the following 6 months. Changes from baseline to 12 months for disease activity and MBDA score showed a significant correlation for methotrexate and prednisolone $(r=0.57, p=0.002)$, methotrexate and placebo $(r=0.57, p=0.001)$ and for all groups $(r=0.56, p<0.001)$. The final prospective study which included 148 patients, showed that MBDA predicted remission in RA and could differentiate between small differences in disease activity (low disease activity vs remission states). Baseline MBDA score discriminated baseline remission, area under the curve (AUC) 0.68-0.75, and intermittent/sustained remission (AUC 0.65-0.74). MBDA also predicted long term remission after one year.

Conclusion: MBDA appears to be a useful tool in day-to-day clinical practice that can be used in the diagnosis, monitoring of treatment and prediction of outcomes of patients with RA. MBDA correlated well with disease activity and response to treatment. It is also a good predictor of long-term disease remission.

Disclosure of Interests: None declared

DOI: 10.1136/annrheumdis-2021-eular.677

\begin{tabular}{|l|l}
\hline AB0106 & ASSOCIATED FACTORS FOR PRESENTING WITH \\
MODERATE TO HIGH DISEASE ACTIVITY ON THE \\
FIRST RHEUMATOLOGY VISIT IN AN OUTPATIENT \\
CLINIC-BASED COHORT OF COLOMBIAN RA \\
PATIENTS: A REAL-WORLD CROSS-SECTIONAL \\
STUDY
\end{tabular}

K. Maldonado-Cañón ${ }^{1,2}$, P. Coral Alvarado ${ }^{2,3}$, P. A. Mendez Patarroyo $o^{2,3}$, E. Bermúdez ${ }^{2}, G$. Quintana Lopez ${ }^{1,2,3}$ on behalf of Reumavance Research Group. ${ }^{1}$ School of Medicine - National University of Colombia, Reumavance Research Group. Internal Medicine Department, Bogotá, Colombia; ${ }^{2}$ Fundacion Santa Fe de Bogota University Hospital, Reumavance Research Group. Internal Medicine Department, Bogotá, Colombia; ${ }^{3}$ School of Medicine Universidad de Los Andes, Reumavance Research Group. Internal Medicine Department, Bogota, Colombia

Background: Rheumatoid arthritis (RA) is characterized by autoantibody formation and expansion such as the rheumatoid factor (RF), anti-citrullinated protein antibodies (ACPA), and antinuclear antibodies (ANA). Autoantibody identification has been considered as crucial not only for the diagnosis but also for its eventual association with the disease activity, to the extent of playing a pivotal role as a poor prognostic factor among patients ${ }^{1}$

Objectives: Our aim was to assess the eventual association between certain clinical-epidemiological factors and presenting with moderate to high disease activity on the first rheumatology visit in an outpatient clinic-based cohort of Colombian RA patients.

Methods: We conducted a cross-sectional analytic study utilizing the database of an outpatient clinic based in the Rheumatology Department at Fundación Santa Fe de Bogotá University Hospital. Data were collected from May 2013 until Sep. 2020 and included age, gender, autoimmune- and non-autoimmune comorbidities, family history of autoimmunity (including RA), smoking and alcoholism personal history, previous DMARD and oral steroid use, nutritional status, disease duration defined as established or early RA (disease duration of $\leq 1$ year before starting a DMARD), autoantibody profile information (RF ACPA, and ANA), and disease activity, assessed by the DAS28- ESR. Multiple logistic regression with backward selection was performed to build the final fitted model.

Results: Data from a total of 1229 patients were included in the analysis. Our population was primarily female $(n=938,76,3 \%)$, the majority had an initial diagnosis of established RA ( $n=952(77,5 \%)$, and presentation on the first consultation with moderate to high disease activity was seen on $65,2 \%$ of the cases $(n=801)$. Being male and having an autoimmune comorbidity were statistically significant associated protective factors whereas strongly positive RF and ACPA levels, positive high ANA titres, and established RA were recognized as risk factors after adjustment for the effects of possible confounders such as age, smoking status, previous DMARD use, and family history of RA. Table 1 provides the complete results obtained from the multiple logistic regression model.

Table 1. Multiple logistic regression analysis's results.

\begin{tabular}{|c|c|c|}
\hline & OR $(95 \% \mathrm{Cl})$ & p-value \\
\hline Male & $0,52(0,39-0,69)$ & $<0,0001$ \\
\hline Age & $0,99(0,99-1)$ & 0,24 \\
\hline Autoimmune Comorbidities & $0,41(0,21-0,80)$ & 0,009 \\
\hline Family History of RA & $0,85(0,61-1,14)$ & 0,26 \\
\hline Current Smoking & $1,26(0,78-2,07)$ & 0,29 \\
\hline History of Smoking & $1,26(0,81-2)$ & 0,30 \\
\hline Previous DMARD use & $0,91(0,70-1,18)$ & 0,45 \\
\hline RF strong titers i.e., > 80 U/ml & $1,56(1,21-2,02)$ & 0,0007 \\
\hline ACPA strong titers i.e., > $80 \mathrm{U} / \mathrm{ml}$ & $1,59(1,19-2,11)$ & 0,0016 \\
\hline ANA low positive titers i.e., < 1:640 dil. & $0,94(0,72-1,12)$ & 0,67 \\
\hline ANA strong titers i.e., > 1:640 dil. & $1,98(1,23-3,27)$ & 0,0063 \\
\hline Disease duration (established RA) & $1,41(1,05-1,90)$ & 0,0277 \\
\hline
\end{tabular}

Bold values indicate statistically significant.

Conclusion: These findings have significant implications for the understanding of the disease activity evaluated on the first contact of a RA patient with a rheumatologist. Our data supports the consideration of being male as a protective factor; however, suggest that autoimmune comorbidities could also condition a lower disease activity for such scenarios, which may be explained by the fact that such patients were referred from- and already being treated by- other practitioners. Additionally, our research provides insights for the consideration of high ANA titers as a potentially poor prognosis factor to be identified as early as on the first visit. ANA's use on daily practice is an intriguing matter which could be usefully explored in further research with distinct methodological approaches.

\section{REFERENCES:}

[1] Smolen JS, Landewé RBM, Bijlsma JWJ, et al. EULAR recommendations for the management of rheumatoid arthritis with synthetic and biological disease-modifying antirheumatic drugs: 2019 update. Annals of the Rheumatic Diseases 2020;79:685-699

Disclosure of Interests: None declared

DOI: 10.1136/annrheumdis-2021-eular.806

AB0107

CHANGES IN EXTRACELLULAR MATRIX BIOMARKER C3M CORRELATE WITH ABATACEPT RESPONSE IN SEROPOSITIVE EARLY RA

S. L. Bridges ${ }^{1}$, J. Buckner ${ }^{2}$, M. A. Maldonado ${ }^{3}$, V. Bykerk ${ }^{4}$, N. Ray ${ }^{5}$,

S. Mukherjee ${ }^{6}$, N. Ohtsuka ${ }^{7}{ }^{1}$ Hospital for Special Surgery, Department of Medicine, New York, United States of America; ${ }^{2}$ Benaroya Research Institute at Virginia Mason, Center for Translational Immunology, Seattle, United States of America; ${ }^{3}$ Bristol Myers Squibb, Clinical R\&D, Immunoscience, Princeton, United States of America; ${ }^{4}$ Hospital for Special Surgery, Rheumatology, New York, United States of America; ${ }^{5}$ Bristol Myers Squibb, Global Drug 